\title{
Nouvelles chambres d'ionisation primaires du LNE-LNHB pour la détermination du kerma dans l'air dans un faisceau de cobalt-60
}

\section{New primary ionization chambers at LNE-LNHB for determining the air kerma in a cobalt-60 beam}

\author{
Frank DELAUNAY, Marc DONOIS, Jean GOURIOU, Eric LEROY et Aimé OSTROWSKY
}

CEA, LIST, Laboratoire national Henri Becquerel (LNE-LNHB), 91191 Gif-sur-Yvette Cedex, France, franck.delaunay@ cea.fr.

\section{Résumé}

La grandeur de référence en radioprotection est le kerma dans l'air. Dans un faisceau de cobalt-60, la référence est obtenue à l'aide d'une chambre d'ionisation à cavité dont le volume est mesuré. La nouvelle référence du LNE-LNHB est basée sur l'utilisation de six chambres différentes, contrairement à la précédente référence qui n'utilisait qu'une seule chambre. Bien que toutes les chambres d'ionisation aient été autant que possible traitées de façon identique (fabrication, mesures de volume, calculs de correction de paroi, corrections sur le courant mesuré) un écart maximum de $0,2 \%$ a été observé entre les débits de kerma dans l'air obtenus avec les six chambres. Le débit de kerma dans l'air dans les conditions de référence est pris égal à la moyenne arithmétique des six débits de kerma dans l'air. Parmi les différents facteurs nécessaires pour calculer le débit de kerma dans l'air, certains sont communs à toutes les chambres d'ionisation à paroi graphite (par exemple, énergie moyenne dépensée par un électron pour produire une paire électron-ion dans l'air $\mathrm{sec}$ ), tandis que d'autres varient pour chaque chambre (par exemple, volume de collection ionique dans la cavité d'air). Considérant que les incertitudes sur les mesures des chambres d'ionisation individuelles semblent légèrement sous-estimées, l'incertitude sur la moyenne des produits des termes dépendant des six chambres d'ionisation est prise égale à l'écart type sur la distribution de ces produits $(0,08 \%)$. Cette nouvelle détermination de débit de kerma dans l'air au cobalt-60 est supérieure de $0,09 \%$ à la référence précédente et l'incertitude diminue de $0,38 \%$ à $0,31 \%$.

MOTS CLÉS : MÉTROLOGIE DES RAYONNEMENTS IONISANTS, KERMA DANS L'AIR, COBALT-60.

\footnotetext{
Abstract

For radioprotection, the reference quantity is air kerma. For an cobalt-60 beam, the reference dosimeter is a cavity ionization chamber
}

whose volume is measured. The new LNE-LNHB reference is based on six different chambers instead of one as was done previously. Although every new ionization chamber was treated as much as possible in the same way (manufacturing, measurements of volumes, wall effect calculations, current corrections), a maximum discrepancy of $0.2 \%$ was observed between the final measurement results from each chamber. The final value of the air kerma rate in reference conditions was determined as the mean value of the measurement results from all six chambers. Among the different factors whose determination is necessary to calculate the air kerma rate, some are considered independent of or common to all the graphite-walled ionization chambers (for example, mean energy expended by an electron to produce an ion pair in dry air), while others vary for each chamber (for example, air cavity ionic collection volume). Considering that the uncertainties of the individual ionizationchamber measurement results seem slightly underestimated, the uncertainty on the mean of the six chamber-dependent factors products was taken equal to the standard deviation of the sample composed of the six chamber-dependent factors products $(0.08 \%)$. Compared to the previous standard, the air kerma rate of the ${ }^{60}$ Co photon beam would then increase by $0.09 \%$ and the air kerma rate uncertainty would drop from $0.38 \%$ to $0.31 \%$. This article describes the procedure used to establish the primary standard in terms of absorbed dose to tissue of LNE-LNHB.

KEY WORDS: IONIZING RADIATION METROLOGY, AIR KERMA, COBALT-60.

\section{Introduction}

Le Laboratoire National Henri Becquerel (LNELNHB) est en charge des références dosimétriques pour les photons et les particules chargées. À ce titre, il a en particulier en charge la référence française en kerma 
dans l'air pour le cobalt-60 utilisée en radioprotection. Le kerma est, pour des particules non directement ionisantes, le quotient de la somme des énergies cinétiques initiales de toutes les particules chargées libérées dans un élément de matière par la masse de cet élément. Aujourd'hui, le kerma n'est plus guère utilisé pour la radiothérapie mais il reste la grandeur de référence pour la radioprotection.

Le dosimètre primaire précédemment utilisé comme référence était une chambre d'ionisation à paroi graphite nommée GCS-10-1. Cette chambre avait été dessinée et construite au laboratoire, il y a environ 25 ans. Sa forme est cylindrique avec deux extrémités hémisphériques. Le volume interne de la cavité d'air est d'environ $9,5 \mathrm{~cm}^{3}$. La dernière comparaison faite avec cette chambre en 2003 avec le Bureau international des poids et mesures (BIPM) avait conduit à un rapport LNE-LNHB/BIPM de $0,9981(27)(k=1)[1]$

Considérant qu'une seule chambre d'ionisation étalon ne pouvait constituer une référence nationale suffisamment sûre et robuste, le LNE-LNHB a décidé de construire six nouvelles chambres d'ionisation à paroi graphite de différentes formes et de différents volumes. Trois d'entre elles sont de forme sphérique et les trois autres sont de forme cylindrique aux extrémités hémisphériques (même forme que la chambre GCS-10-1) [2].

Dans cet article, toutes les incertitudes sont données à un écart type $(k=1)$.

\section{Rappel : principe d'une mesure de kerma dans l'air avec une chambre d'ionisation étalon à cavité}

La mesure du kerma dans l'air dans des faisceaux de photons $\gamma$ de haute énergie est réalisée à l'aide de chambres d'ionisation étalons à cavité. Ces chambres sont constituées, de l'extérieur vers l'intérieur, d'une paroi externe faisant office d'électrode, d'une cavité remplie de gaz (air), et d'une électrode centrale. L'application d'une haute tension à ces électrodes crée, à l'intérieur de la cavité, un champ électrique qui permet de collecter les charges résultant de l'ionisation de l'air sous l'effet du rayonnement. Le courant d'ionisation résultant est proportionnel au débit de la dose déposée dans la cavité. Celui-ci est en général mesuré en suivant la charge d'un condensateur de capacité connue (méthode à « taux de dérive »).

Les chambres utilisées pour la mesure du kerma dans l'air dans un faisceau de cobalt-60 ont généralement une paroi externe en graphite, matériau dont le numéro atomique est proche de celui de l'air. Leur cavité est en communication avec l'extérieur. Les caractéristiques de l'air présent dans cette cavité (pression, température, humidité relative) suivent donc celles de l'air ambiant.

L'état de l'art pour la fabrication des chambres à paroi graphite, les principes de base et les théories qui s'y rapportent ainsi que les termes correctifs pour le calcul du kerma dans l'air sont décrits dans l'article de Büermann et Burns [3] et ne sont pas répétés ici.

Le débit de kerma dans l'air ( $\left.\dot{K}_{\text {air }}\right)$, en un point et à une date de référence donnés, est déterminé en utilisant l'équation suivante :

$$
\begin{aligned}
& \dot{K}_{\text {air }}=\frac{\Pi}{\rho} \frac{W}{\mathrm{e}} S_{\text {wall, air }}\left(\frac{\mu_{\mathrm{en}}}{\rho}\right)_{\text {air, wall }} \frac{1}{1-\bar{g}_{\text {air }}} \\
& \text { avec } \Pi=\left(I k_{s} k_{\text {pol }} k_{\mathrm{st}} k_{\mathrm{an}} k_{\mathrm{rn}} k_{\text {wall }} k_{\text {cel }}\right) / V_{\text {col }}
\end{aligned}
$$

$I$ courant mesuré corrigé avec les facteurs $k_{T}, k_{p}, k_{h}$, $k_{C}, k_{\text {att }}, k_{t}$ et $k_{d}$. Les définitions des différents facteurs de correction sont données plus loin dans l'article, seuls les termes les plus importants sont expliqués ci-après :

- $\rho V_{\text {col }}=m$, masse d'air dans le volume de collection ionique de la cavité ( $\left.V_{\mathrm{col}}\right)$, avec $\rho$ masse volumique de l'air de la cavité dans les conditions de référence;

- $W$, énergie moyenne dépensée par un électron de charge e pour produire une paire électron-ion dans de l'air sec ;

- $S_{\text {wall, air, rapport des pouvoirs de ralentissement }}$ moyen des électrons dans le milieu de l'électrode externe et dans l'air sec;

- $\left(\mu_{\mathrm{en}} / \rho\right)_{\text {air, wall }}$, rapport des coefficients moyens d'absorption d'énergie massique dans l'air et dans le milieu de l'électrode externe;

- $\bar{g}_{\text {air }}$, fraction moyenne d'énergie cinétique des électrons perdue sous forme de rayonnement de freinage ;

- $k_{\text {wall }}$, facteur de correction de perturbation dû à l'atténuation de la fluence et à la diffusion des photons dans la paroi de la chambre.

\section{Nouveaux dosimètres primaires pour le kerma dans l'air}

\subsection{Description des nouvelles chambres d'ionisation}

Les plans, le choix des matériaux et l'assemblage des pièces ont été faits par le LNE-LNHB. Trois des chambres sont de forme sphérique et les trois autres sont cylindriques avec extrémités hémisphériques, qualifiées simplement de cylindriques dans la suite de l'article. Des exemples de ces deux types de chambre sont présentés sur la figure 1. Un même rayon interne de cavité est utilisé à la fois pour une chambre sphérique et pour une chambre cylindrique. Ces rayons internes sont de $1,0 \mathrm{~cm}, 1,1 \mathrm{~cm}$ et $1,2 \mathrm{~cm}$. Pour les chambres cylindriques, la hauteur de la partie cylindrique est égale au rayon interne de la cavité. Les parois des chambres sont en graphite.

L'épaisseur radiale de la paroi graphite (électrode externe) est de $3 \mathrm{~mm}$, suffisante pour atteindre l'équilibre électronique dans un faisceau de cobalt-60. La masse volumique du graphite est de $1,847(18) \mathrm{g} \cdot \mathrm{cm}^{-3}$. L'électrode interne est constituée du même graphite. La forme 


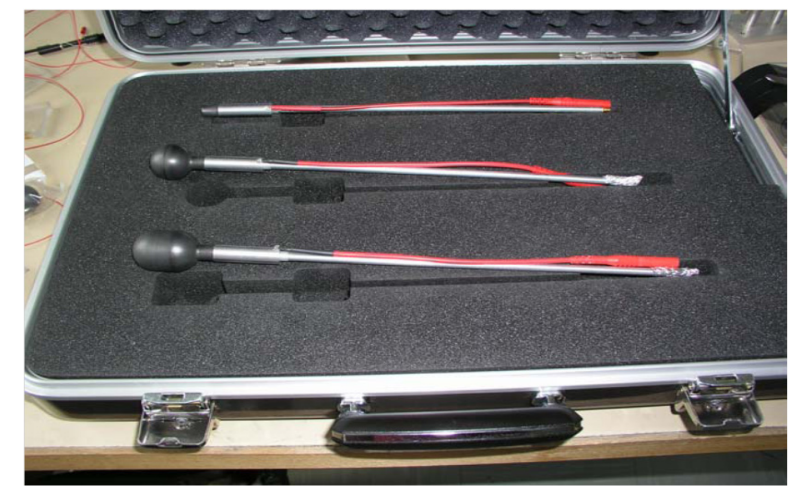

Fig. 1. - Photographie (du haut vers le bas) du manche factice, de la plus grosse chambre sphérique et de la plus grosse chambre cylindrique des nouvelles chambres d'ionisation du LNE-LNHB.

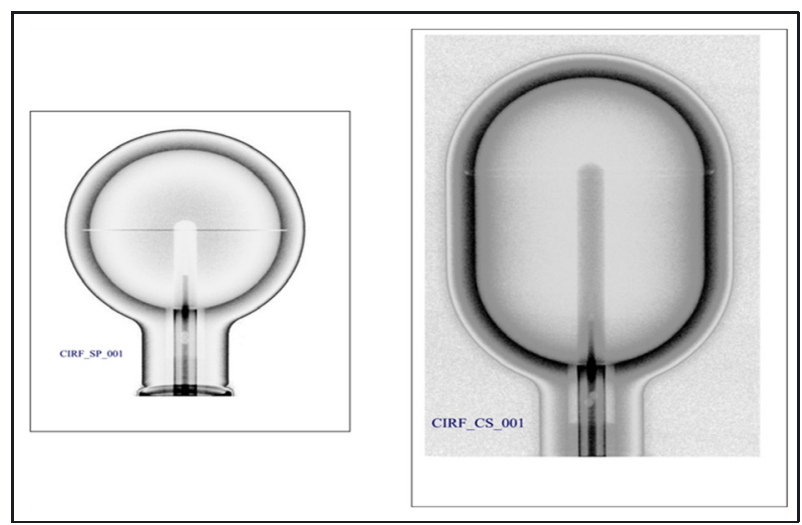

Fig. 2. - Radiographie de la plus grosse chambre d'ionisation sphérique et de la plus grosse chambre d'ionisation cylindrique (la ligne noire visible à l'équateur de la chambre sphérique est due à un défaut d'assemblage entre les deux hémisphères et est incluse dans les mesures du volume de la cavité).

de cette électrode est cylindrique avec un sommet hémisphérique et son diamètre est de $3 \mathrm{~mm}$. Les bases des extrémités hémisphériques des électrodes (interne et externe) sont dans le même plan (fig. 2). Chaque cavité, délimitée par l'électrode externe en graphite, peut être visualisée comme une sphère (ou un cylindre prolongé de deux hémisphères) et un col par lequel l'électrode interne et le manche sont fixés. Le bord interne de l'électrode externe (limite entre l'hémisphère bas et le col dans la cavité) est légèrement limé afin d'éviter les bords abrupts dans la cavité. Quatre capots additionnels pouvant être placés autour de l'électrode externe ont été fabriqués pour les chambres les plus grandes (sphérique et cylindrique) afin de tester les calculs de Monte-Carlo d'effet de paroi. Ces capots permettent d'obtenir des épaisseurs de paroi de $5 \mathrm{~mm}$ et $7 \mathrm{~mm}$ respectivement.

L'anneau de garde est en Dural. La figure 3 illustre les résultats du calcul du champ électrique dans le bas de la cavité avec l'anneau de garde placé à $0,2 \mathrm{~mm}$ en dessous de l'électrode interne. Le rayon externe de l'anneau de garde et celui de l'électrode interne sont égaux. Le plan (P) défini par le bord interne de l'électrode externe

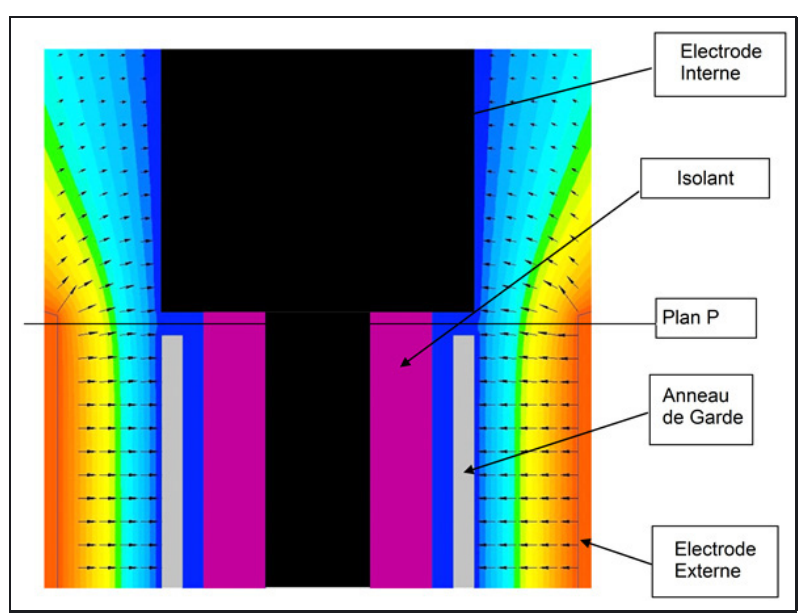

Fig. 3. - Simulation de la force du champ électrique dans le bas de la cavité de la chambre d'ionisation (l'électrode interne en graphite et le câble de collection en Dural juste en dessous sont en noir, l'isolant en polystyrène réticulé en rose, l'électrode de garde en Dural en gris, l'électrode externe en graphite en orange, l'air à l'intérieur de la cavité est représenté par une palette de couleurs du jaune orangé au bleu foncé suivant le potentiel électrique).

est en principe juste au milieu entre le bas de l'électrode interne et le haut de l'anneau de garde. L'isolant est en polystyrène réticulé qui présente une bonne résistance aux dégâts d'irradiation. Un soin particulier a été apporté pour s'assurer que les surfaces de l'isolant sont bien propres et lisses. Afin de réduire les effets de surface entre l'isolant et l'anneau de garde, un espace d'air, d'épaisseur $0,1 \mathrm{~mm}$, sépare sur une longueur de $5 \mathrm{~mm}$ le sommet de l'anneau de garde et l'isolant. L'équilibre entre la pression atmosphérique à l'intérieur et à l'extérieur de la cavité se fait par l'intermédiaire de deux trous placés symétriquement dans la partie graphite du manche. Un manche factice, identique au vrai, permet de mesurer l'excès de signal dû au rayonnement diffusé dans le manche de la chambre d'ionisation (fig. 1).

La haute tension est appliquée sur l'électrode externe tandis que l'électromètre est connecté à l'électrode interne. L'anneau de garde est à la même tension que l'électrode interne.

\subsection{Détermination du volume de la cavité}

La détermination du volume de collection dans la cavité $\left(V_{\text {col }}\right)$ est l'une des principales étapes de la caractérisation d'une chambre d'ionisation à cavité. Le volume de collection d'une chambre d'ionisation primaire doit être déterminé par des méthodes de mesure de volume, sans irradiation, ni mesure du signal de la chambre d'ionisation. Le volume de collection est défini comme le volume dans la cavité où les particules chargées créées (électrons et ions négatifs ou ions positifs suivant la polarité) sont collectées par l'électrode centrale et leur charge totale mesurée. 
Tableau 1

Volumes estimés des différentes chambres d'ionisation.

\begin{tabular}{|c|c|c|c|c|c|c|c|}
\hline & $\begin{array}{c}\text { Rayon } \\
\text { interne } \\
(\mathrm{cm})\end{array}$ & $\begin{array}{c}V_{\text {outer }} \\
\left(\mathrm{cm}^{3}\right)\end{array}$ & $\begin{array}{c}V_{\text {neck }} \\
\left(\mathrm{cm}^{3}\right)\end{array}$ & $\begin{array}{c}V_{\text {inner }} \\
\left(\mathrm{cm}^{3}\right)\end{array}$ & $\begin{array}{c}V_{0} \\
\left(\mathrm{~cm}^{3}\right)\end{array}$ & $\begin{array}{c}V_{\text {edge }} \\
\left(\mathrm{cm}^{3}\right)\end{array}$ & $\begin{array}{c}V_{\text {col }} \\
\left(\mathrm{cm}^{3}\right)\end{array}$ \\
\hline \multirow{3}{*}{ Cylindrique } & 1,2 & $13,0869(15)$ & 0,4426 & 0,17349 & 0,00069 & 0,00008 & $12,4701(20)$ \\
\cline { 2 - 8 } & 1,1 & $10,4605(8)$ & 0,4431 & 0,15926 & 0,00069 & 0,00008 & $9,8574(15)$ \\
\cline { 2 - 8 } & 1,0 & $7,7632(9)$ & 0,4429 & 0,14518 & 0,00063 & 0,00008 & $7,1745(15)$ \\
\hline \multirow{3}{*}{ Sphérique } & 1,2 & $7,7475(17)$ & 0,4419 & 0,08936 & 0,00074 & 0,00008 & $7,2154(21)$ \\
\cline { 2 - 8 } & 1,1 & $5,9924(19)$ & 0,4417 & 0,08224 & 0,00076 & 0,00008 & $5,4676(23)$ \\
\cline { 2 - 8 } & 1,0 & $4,6254(8)$ & 0,4425 & 0,07517 & 0,00079 & 0,00008 & $4,1069(15)$ \\
\hline
\end{tabular}

La séparation entre le volume de collection et le volume de non-collection est supposé correspondre au plan (P) situé au milieu entre le bas de l'électrode interne et le haut de l'anneau de garde (fig. 3). Il est supposé, qu'au-dessus de ce plan les particules chargées de la bonne polarité sont collectées par l'électrode centrale, tandis qu'en dessous, elles sont collectées par l'anneau de garde. Dans les deux cas, les particules chargées de l'autre polarité, se dirigent vers l'électrode externe. Bien entendu, comme certaines particules chargées pénètrent dans la cavité avec une vitesse non nulle, ce n'est pas aussi simple. Il est alors supposé qu'il existe un certain équilibre et que le nombre de particules chargées d'une polarité qui traversent ce plan dans une direction est égal au nombre de particules chargées de même polarité qui traversent ce plan dans l'autre direction. Comme il n'y a pas de champ électrique entre l'électrode interne et l'anneau de garde, il faut retrancher au volume de collection, l'espace entre l'électrode interne et le plan (P).

Les mesures conduisant à la détermination du volume de collection ont été effectuées au Laboratoire national de métrologie et d'essais (LNE). Le volume complet à l'intérieur de l'électrode externe (cavité de collection et col, $\left.V_{\text {outer }}\right)$ a été mesuré par pesées après remplissage avec de l'eau. Les volumes du col ( $\left.V_{\text {neck }}\right)$, de l'électrode centrale $\left(V_{\text {inner }}\right)$ et le volume $\left(V_{0}\right)$ entre le plan $(\mathrm{P})$ et l'électrode centrale ont été déterminés à partir de mesures de longueurs et de diamètres. Le volume de la cavité $\left(V_{\text {edge }}\right)$ créé par le limage de la paroi pour éviter les bords abrupts est considéré ne contribuer que pour moitié au volume de collection, et il est très petit $\left(2 \times 10^{-5}<\right.$ $\left.V_{\text {edge }}<18 \times 10^{-5} \mathrm{~cm}^{3}\right)$. L'incertitude sur l'enfoncement de l'électrode centrale dans la cavité est estimée augmenter l'incertitude totale sur le volume de collection d'une valeur égale à $V_{0}$. Le volume de collection est calculé comme suit :

$$
V_{\text {col }}=V_{\text {outer }}-V_{\text {neck }}-V_{\text {inner }}-V_{0}-1 / 2 V_{\text {edge }} .
$$

Le tableau 1 donne les valeurs estimées des différents volumes.

\subsection{Mesures préliminaires}

La haute tension de travail de la précédente chambre d'ionisation primaire (GCS-10-1) est de 800 V. Les cou- rants ioniques produits dans un prototype des nouvelles chambres d'ionisation ont été mesurés dans un faisceau de cobalt-60 à différents débits $\left(175 \mu \mathrm{Gy} \cdot \mathrm{s}^{-1}\right.$ à $\left.40 \mathrm{mGy} \cdot \mathrm{s}^{-1}\right)$ et à différentes hautes tensions $(0$ à $1000 \mathrm{~V}$ par pas de $50 \mathrm{~V}$ ) afin de déterminer une bonne tension de travail. Les courants mesurés pour $850 \mathrm{~V}$ et $450 \mathrm{~V}$ différaient de moins de $0,1 \%$ pour chaque débit de dose testé. La valeur choisie pour la tension de travail est de $850 \mathrm{~V}$ pour toutes les nouvelles chambres d'ionisation. Les rapports signal à bruit dans un faisceau de $10 \mathrm{mGy} \cdot \mathrm{s}^{-1}$ sont compris entre $1 \times 10^{5}$ and $4 \times 10^{5}$ pour les nouvelles chambres (à comparer à $2 \times 10^{5}$ pour la GCS-10-1). Dans un faisceau 10 fois moins intense, les rapports sont compris entre $1,5 \times 10^{4}$ et $1 \times 10^{5}$ (à comparer à $1 \times 10^{5}$ pour la GCS-10-1). Le courant de fuite devrait donc être négligeable pour les mesures.

\section{Facteurs correctifs}

L'expression permettant d'obtenir le débit de kerma dans l'air (1) est constituée de termes qui dépendent soit exclusivement des caractéristiques du faisceau sans le détecteur, soit exclusivement des caractéristiques du détecteur $\left(V_{\mathrm{col}}\right)$ ou de l'interaction entre le faisceau, l'environnement du faisceau et le détecteur $\left(I k_{s} k_{\mathrm{pol}} k_{\mathrm{st}} k_{\mathrm{an}} k_{\mathrm{rn}} k_{\mathrm{wall}} k_{\mathrm{cel}} / \rho\right)$. Dans cette partie de l'article, seule la dernière catégorie sera discutée. Ceci correspond au signal mesuré (courant d'ionisation) et à ses corrections, à la masse volumique de l'air de la cavité et aux effets de perturbations dus à la présence du détecteur. Les autres termes et leurs incertitudes sont identiques à ceux utilisés pour la chambre GCS-10-1 [4]. Puisque l'un des buts de cet article est de comparer entre eux les résultats obtenus avec les six chambres d'ionisation, les incertitudes communes ne sont prises en compte qu'ultérieurement.

\subsection{Corrections atmosphériques $\left(k_{T}, k_{p}, k_{h}\right)$}

Différents facteurs correctifs sont utilisés pour obtenir le courant qui serait mesuré dans les conditions atmosphériques de référence : température 293,15 K, pression $1013,25 \mathrm{hPa}$ et humidité relative $0 \%$ (air sec). Il est alors possible d'utiliser la masse volumique de l'air dans les conditions normales pour calculer la masse d'air dans la 
cavité. Le facteur de correction d'humidité $\left(k_{h}\right)$ provient de l'ICRU 31 [5]. Les facteurs de correction avec leurs incertitudes sont :

$$
\begin{array}{rlrl}
k_{T} & =T(\mathrm{~K}) / 293,15 & u\left(k_{T}\right) / k_{T} & =0,051 \% \\
k_{p} & =1013,25 / p(\mathrm{hPa}) & u\left(k_{p}\right) / k_{p} & =0,017 \% \\
k_{h} & =0,997 & u\left(k_{h}\right) / k_{h} & =0,030 \% .
\end{array}
$$

\subsection{Corrections électriques $\left(k_{\mathrm{pol}}, k_{s}, k_{C}\right)$}

Des explications sur les facteurs correctifs liés à la recombinaison $k_{s}$ et à la polarité $k_{\mathrm{pol}}$ ainsi que les références aux travaux originaux peuvent être trouvées dans un article rédigé par Boag [6].

Le courant mesuré dépend de la polarité de la haute tension. Afin de corriger, partiellement, de cet effet, le facteur de correction de polarité est appliqué au courant mesuré $(I)$,

$$
\begin{aligned}
& k_{\mathrm{pol}}=\left(I_{+}-I_{-}\right) / 2 I \\
& u\left(k_{\mathrm{pol}}\right) / k_{\mathrm{pol}}=0,0088 \% .
\end{aligned}
$$

Pour une haute tension de $+850 \mathrm{~V}$ appliquée à l'électrode externe, le facteur de correction de polarité est compris entre 1,000 18 et 1,000 26 pour les nouvelles chambres d'ionisation sphériques et entre 1,000 35 et 1,000 43 pour les nouvelles chambres d'ionisation cylindriques (y compris la GCS-10-1). Une faible correction de polarité est un bon indicateur de la qualité d'une chambre d'ionisation.

Une partie des charges créées dans le volume de collection n'est pas collectée à cause de la recombinaison. Le facteur de correction de recombinaison peut être factorisé en un terme de recombinaison initiale (au sein de la même trace, indépendant du débit de dose) et un terme de recombinaison en volume (impliquant des ions produits dans différentes traces, dépendant du débit de dose). Le facteur de correction de recombinaison peut se calculer à l'aide de la formule suivante

$$
k_{s} \approx 1+A /|V|+B / V^{2}|I|
$$

avec $V$ haute tension appliquée, $I$ courant mesuré, $A$ et $B$ constantes pour une chambre d'ionisation donnée dans un faisceau donné. Si des mesures $\left(I_{V}, I_{V / m}\right)$ à deux différentes tensions $(\mathrm{V}, \mathrm{V} / \mathrm{m})$ sont faites à différentes distances de la source, la courbe du rapport des courants mesurés à la même distance $\left(I_{V} / I_{V / m}\right)$ tracé en fonction de la valeur du courant $\left(\left|k_{\mathrm{pol}} I_{V}\right|\right)$ est une droite [7]. La pente de la droite est égale à $\left(m^{2}-1\right) B / V^{2}$ et l'ordonnée à l'origine à $1+(m-1) A / V$. Les valeurs de $A$ et de $B$ ont été déterminées pour chaque nouvelle chambre d'ionisation. Puisque la quantité $(1+A / V)$ correspond au facteur de correction de recombinaison initiale, il a été comparé au résultat obtenu à l'aide de la méthode « classique »,

$$
k_{s}=1+\frac{I_{V} / I_{V / m}-1}{m-I_{V} / I_{V / m}},
$$

Tableau 2

Facteurs de correction de recombinaison avec leurs incertitudes de type A.

\begin{tabular}{|c|c|c|c|c|}
\hline & $\begin{array}{c}\text { Rayon } \\
\text { interne } \\
(\mathrm{cm})\end{array}$ & $1+A / V$ & $\begin{array}{c}k_{s} \\
\text { (Bas débit) }\end{array}$ & $\begin{array}{c}k_{s} \\
\text { Exemples }\end{array}$ \\
\hline \multirow{3}{*}{ Cylindrique } & 1,2 & $1,00050(3)$ & $1,00039(31)$ & $1,00087(22)$ \\
\cline { 2 - 6 } & 1,1 & $1,00088(2)$ & $1,00109(18)$ & $1,00118(20)$ \\
\cline { 2 - 5 } Sphérique & 1,0 & $1,00045(10)$ & $1,00057(19)$ & $1,00058(11)$ \\
\hline & 1,2 & $1,00075(5)$ & $1,00070(17)$ & $1,00124(35)$ \\
\cline { 2 - 5 } & 1,1 & $1,00062(2)$ & $1,00053(16)$ & $1,00094(22)$ \\
\cline { 2 - 5 } & 1,0 & $1,00050(2)$ & $1,00066(37)$ & $1,00068(11)$ \\
\hline
\end{tabular}

dans un faisceau de cobalt-60 de bas débit où la recombinaison initiale est prédominante. Les résultats sont donnés dans le tableau 2 et sont en accord à mieux que $0,02 \%$. Le débit de dose était d'environ $33 \mathrm{~Gy} \cdot \mathrm{h}^{-1}$ au début de cette étude et d'environ $25 \mathrm{~Gy} \cdot \mathrm{h}^{-1}$ à la fin. La même haute tension de $850 \mathrm{~V}$ étant appliquée pour toutes les chambres, plus le rayon interne de la chambre est grand, plus le champ électrique est petit et plus le facteur de correction de recombinaison est élevé. L'incertitude sur $k_{s}$ pour les plus forts débits de dose est plus grande que l'incertitude sur $(1+A / V)$, mais ce pourrait ne pas être toujours le cas puisque $A$ et $B$ sont déterminés à partir des mêmes données et ne sont donc pas indépendants.

Plusieurs facteurs de correction sont appliqués au condensateur permettant de mesurer la charge :

$$
\begin{aligned}
& C=C_{\text {cal }} k_{\text {freq }} k_{\text {temp }} k_{\text {humid }} k_{\text {drift }}+C_{\text {paras }} \\
& u\left(k_{C}\right) / k_{C}=0,089 \%,
\end{aligned}
$$

avec $C_{\text {cal }}$ capacité du condensateur et $C_{\text {paras }}$ capacité interne de la chaîne de mesure. Les autres termes corrigent des différences entre les conditions d'étalonnage du condensateur et les conditions d'utilisation : $k_{\text {freq }}$ (étalonnage à $1000 \mathrm{~Hz}$, mesures à $0 \mathrm{~Hz}$ ), $k_{\text {temp }}$ (température du condensateur), $k_{\text {humid }}$ (humidité de l'air) et $k_{\text {drift }}$ (dérive du condensateur entre deux étalonnages).

\subsection{Corrections des conditions de référence $\left(k_{\mathrm{att}}, k_{t}, k_{d}\right)$}

Le facteur de correction d'atténuation $\left(k_{\text {att }}\right)$ compense de la différence d'atténuation du faisceau entre la source et le détecteur placé au point de référence dans les conditions atmosphériques réelles et dans les conditions de référence. Avec $d$ la distance entre la source et le détecteur, $\rho_{0}$ la masse volumique d'air dans les conditions de référence, $\mu / \rho$ le coefficient d'atténuation massique de l'air,

$$
\begin{aligned}
k_{\text {att }} & =\exp \left(\frac{\mu}{\rho} \rho_{0} d\left(\frac{1}{k_{T} k_{p}}-1\right)\right) \text { à } 1 \mathrm{~m}, \\
u\left(k_{\text {att }}\right) / k_{\text {att }} & =0,0007 \% .
\end{aligned}
$$

Le facteur de correction de décroissance d'activité de la source radioactive $\left(k_{t}\right)$ dépend de l'intervalle de temps 
entre les mesures $(t)$ et la date de référence $\left(t_{0}\right)$. La période de décroissance radioactive $\left(T_{1 / 2}\right)$ provient de [8] :

$$
\begin{aligned}
& k_{t}=\exp \left(\frac{\ln (2)}{T_{1 / 2}}\left(t-t_{0}\right)\right) \text { avec } T_{1 / 2}=5,2711(8) \mathrm{ans}, \\
& u\left(k_{t}\right) / k_{t}=0,0067 \% .
\end{aligned}
$$

Le facteur de correction de distance $\left(k_{d}\right)$ tient compte de l'incertitude sur le positionnement du détecteur en termes de distance à la source,

$$
\begin{aligned}
& k_{d}=1 \quad \text { à } 1 \mathrm{~m}, \\
& u\left(k_{d}\right) / k_{d}=0,016 \% .
\end{aligned}
$$

\subsection{Corrections de perturbation liées au détecteur $\left(k_{\mathrm{st}}, k_{\mathrm{an}}, k_{\mathrm{rn}}, k_{\mathrm{wall}}, \boldsymbol{k}_{\mathrm{cel}}\right)$}

Le facteur de correction de manche $\left(k_{\mathrm{st}}\right)$ soustrait du signal mesuré la contribution due au rayonnement diffusé par le manche de la chambre. Cet effet est mesuré en plaçant un manche factice, identique à celui de la chambre, symétriquement à celui-ci. $k_{\mathrm{st}}$ est compris entre 0,9988 et 0,9991 pour les chambres sphériques, et entre 0,9990 et 0,9993 pour les chambres cylindriques avec une incertitude relative $s\left(k_{\mathrm{st}}\right) / k_{\mathrm{st}}=0,02 \%$.

La référence en kerma dans l'air est définie en un point mais les mesures du dosimètre sont faites sur un volume fini. La correction d'anisotropie corrige le courant mesuré pour l'amener à la valeur qu'il aurait si la fluence énergétique du faisceau (sans dosimètre présent) était homogène sur le volume du dosimètre et égale à celle au point de référence. Ce facteur correctif est factorisé en deux termes, pour les non-uniformités axiale $\left(k_{\text {an }}\right)$ et radiale $\left(k_{\mathrm{rn}}\right)$. Le facteur de correction de non-uniformité axiale est pris égal à l'unité $[9,10]$ sans aucune incertitude pour le moment. Le facteur de correction de nonuniformité radiale est déterminé à partir de mesures faites à l'aide d'une petite chambre d'ionisation dans le plan de référence. Pour les chambres cylindriques, les valeurs de $k_{\mathrm{rn}}$ sont comprises entre 1,000 33 et 1,000 44, alors que pour les chambres sphériques, les valeurs de $k_{\mathrm{rn}}$ sont comprises entre 1,000 18 et 1,000 27. L'incertitude relative sur $k_{\mathrm{rn}} s\left(k_{\mathrm{rn}}\right) / k_{\mathrm{rn}}$ est égale à $0,009 \%$.

La correction de paroi $\left(k_{\mathrm{wall}}\right)$ corrige de la perturbation apportée par la paroi de la chambre. Cette correction a été calculée par les codes Monte-Carlo EGSnrc v3 [11] et PENELOPE 2001 [12] selon la méthodologie publiée par Bielajew [13]. Les résultats des calculs sont donnés dans le tableau 3

La différence maximale observée entre les résultats des deux codes est de $0,10 \%$ pour une incertitude de type A de 0,12\%. Les mesures avec les capots additionnels et les calculs des facteurs de correction de paroi correspondants ont été faits pour deux chambres d'ionisation. Le courant corrigé des conditions atmosphériques et multiplié par le facteur de correction d'effet de paroi devrait être constant, indépendant des épaisseurs de paroi. Les rapports avec et sans les capots additionnels sont
Tableau 3

Valeurs calculées de $1 / k_{\text {wall }}$ avec leurs incertitudes de type A en fonction du code utilisé ainsi que le rapport de leurs résultats. CSp fait référence aux chambres cylindriques tandis que $\mathrm{Sp}$ signifie chambre sphérique.

\begin{tabular}{|c|c|c|c|c|}
\hline & $\begin{array}{c}\text { Rayon } \\
\text { interne } \\
\text { de la } \\
\text { cavité } \\
(\mathrm{cm})\end{array}$ & EGSnrc & PENELOPE & Rapport \\
\hline CSp01 & 1,2 & $0,98263(13)$ & $0,9818(10)$ & $1,0009(11)$ \\
\hline $\begin{array}{c}\text { + capot } \\
\text { 2 mm }\end{array}$ & & $0,97128(34)$ & $0,9722(10)$ & $0,9991(11)$ \\
\hline $\begin{array}{c}\text { + capot } \\
\text { 4 mm }\end{array}$ & & $0,96107(17)$ & $0,9614(15)$ & $0,9997(15)$ \\
\hline CSp02 & 1,1 & $0,98309(20)$ & $0,9837(6)$ & $0,9994(6)$ \\
\hline CSp03 & 1,0 & $0,98357(18)$ & $0,9841(6)$ & $0,9995(6)$ \\
\hline Sp01 & 1,2 & $0,98032(7)$ & $0,9812(11)$ & $0,9991(12)$ \\
\hline $\begin{array}{c}\text { capot } \\
2 \mathrm{~mm}\end{array}$ & & $0,96778(43)$ & $0,9687(10)$ & $0,9990(12)$ \\
\hline $\begin{array}{c}\text { + capot } \\
\text { 4 mm }\end{array}$ & & $0,95694(64)$ & $0,9564(15)$ & $1,0006(17)$ \\
\hline Sp02 & 1,1 & $0,98107(23)$ & $0,9803(6)$ & $1,0008(7)$ \\
\hline Sp03 & 1,0 & $0,98148(13)$ & $0,9811(6)$ & $1,0004(6)$ \\
\hline
\end{tabular}

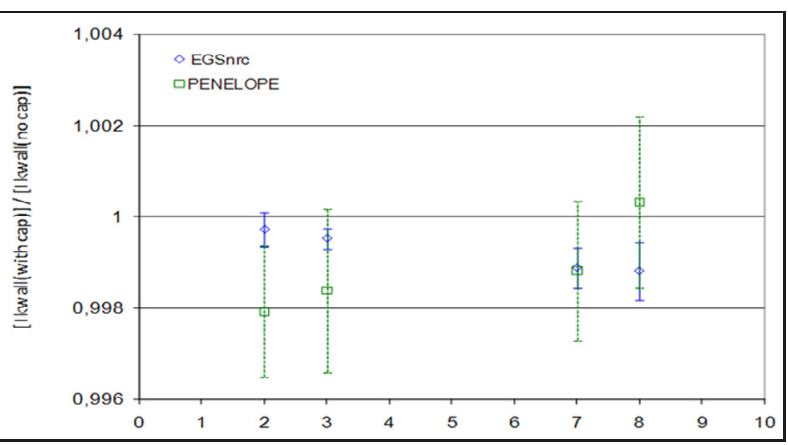

Fig. 4. - Rapports de «I/ $k_{\text {wall }} »$ avec les capots additionnels normalisés à la valeur sans capot additionnel pour une chambre cylindrique (les points d'abscisses 2 et 3 correspondent respectivement aux capots de $2 \mathrm{~mm}$ et $4 \mathrm{~mm}$ ) et pour une chambre sphérique (les points d'abscisses 7 et 8 correspondent respectivement aux capots de $2 \mathrm{~mm}$ et $4 \mathrm{~mm}$ ).

montrés sur la figure 4 pour la plus grosse chambre cylindrique ainsi que la plus grosse chambre sphérique. Les incertitudes associées au code PENELOPE sont plus importantes que celles associées au code EGSnrc mais les conditions de simulation ne sont pas les mêmes (énergies de coupure par exemple). Puisque les résultats des deux codes sont en bon accord, la valeur de $k_{\text {wall }}$ obtenue avec l'incertitude de type A la plus faible est utilisée. L'incertitude sur $k_{\text {wall }}$ est prise comme l'incertitude de type A correspondante ou la différence entre les résultats des deux codes si cette différence s'avère plus grande. Cette méthode donne des incertitudes supérieures à celles qui seraient obtenues avec une moyenne pondérée. Les incertitudes sur $k_{\text {wall }}$ varient entre $0,03 \%$ et $0,07 \%$.

Puisque l'électrode centrale est constituée du même matériau que l'électrode externe, le facteur de perturbation $\left(k_{\mathrm{cel}}\right)$ est pris égal à l'unité. 


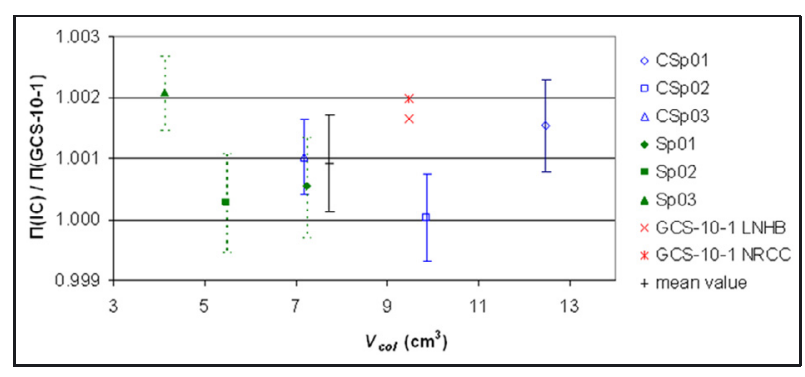

Fig. 5. - Produits des facteurs dépendant des chambres d'ionisation $\Pi=\left(I k_{s} k_{\mathrm{pol}} k_{\mathrm{st}} k_{\mathrm{an}} k_{\mathrm{rn}} k_{\mathrm{wall}} k_{\mathrm{cel}}\right) / V_{\text {col }}$ pour les six nouvelles chambres d'ionisation, ainsi que la valeur moyenne correspondante, normalisés à $\Pi$ (GCS-10-1) en fonction du volume de collection.

\section{Discussion et conclusion}

Le courant dans les conditions de référence, $I$, défini comme le courant mesuré corrigé de $k_{T}, k_{p}, k_{h}, k_{C}, k_{\text {att }}, k_{t}$ et $k_{d}$ a été mesuré pour chaque chambre d'ionisation.

Les produits $(\Pi)$ de tous les facteurs dépendant des chambres

$$
\Pi=\left(I k_{s} k_{\mathrm{pol}} k_{\mathrm{st}} k_{\mathrm{an}} k_{\mathrm{rn}} k_{\mathrm{wall}} k_{\mathrm{cel}}\right) / V_{\mathrm{col}}
$$

ont été comparés entre eux. Ces résultats, normalisés à la GCS-10-1, sont présentés sur la figure 5 en fonction du volume de la cavité. La valeur unité correspond à la chambre GCS-10-1 avec le facteur de correction de paroi déterminé analytiquement. L'incertitude sur la chambre GSC-10-1 n'est pas prise en compte puisque le but est de comparer les nouvelles chambres les unes aux autres. L'incertitude varie entre $0,069 \%$ et $0,082 \%$, suivant la chambre d'ionisation. Les deux points sans barre d'incertitude correspondent à la GCS-10-1 en utilisant des calculs de Monte-Carlo de la correction d'effet de paroi ; calculs faits soit par le LNE-LNHB (valeur moyenne des calculs de PENELOPE et d'EGSnrc) soit par Iwan Kawrakow (alors au NRCC) avec EGSnrc. La dénomination « $\mathrm{CSp} »$ correspond aux chambres cylindriques et la dénomination « $\mathrm{Sp}$ » aux chambres sphériques.

Bien que toutes les chambres d'ionisation aient été traitées autant que possible de la même façon (fabrication, mesures de volume, calcul d'effet de paroi, corrections de courant), les valeurs des produits $\Pi$ obtenus pour les six chambres ne sont pas en parfait accord à un écart-type. La différence maximale observée est de $0,2 \%$ (fig. 5). La valeur moyenne de $\Pi$ pour les six nouvelles chambres d'ionisation, $\bar{\Pi}$, est utilisée pour déterminer le débit de kerma dans l'air du faisceau dans les conditions de référence suivant la formule

$$
\dot{K}_{\text {air }}=\frac{\bar{\Pi}}{\rho} \frac{W}{\mathrm{e}} S_{\text {wall, air }}\left(\frac{\mu_{\mathrm{en}}}{\rho}\right)_{\text {air, wall }} \frac{1}{1-\bar{g}_{\text {air }}} .
$$

Considérant que les incertitudes sur les valeurs de $\Pi$ individuelles sont probablement sous-estimées, l'incertitude adoptée pour la valeur moyenne $\bar{\Pi}$ est l'écart type de la distribution des valeurs de $\Pi$ des six chambres $(0,08 \%)$. Cette valeur peut être comparée à celle obtenue pour la
GCS-10-1 : 0,26\% dont 0,21\% pour le facteur de correction d'effet de paroi. Dans le futur, des améliorations pourraient être recherchées pour mesurer les volumes des cavités et leurs incertitudes. Les incertitudes de type B sur les méthodes utilisées pour déterminer les différents facteurs correctifs peuvent maintenant être ajoutées : $0,02 \%$ pour chacune des corrections suivantes $k_{\mathrm{pol}}, k_{s}, k_{\mathrm{st}}, k_{\mathrm{rn}}$, puis $0,05 \%$ pour $k_{\text {an }}$ et $0,1 \%$ pour $k_{\text {wall }}$.

À l'exception des chambres $\mathrm{Sp} 03$ et CSp01, les valeurs de $\Pi$ sont plus petites que celle correspondant à la GCS- $10^{-1}$ en utilisant le facteur de correction d'effet de paroi calculé par Monte-Carlo mais elles sont toutes supérieures à la valeur de $\Pi$ correspondant à la GCS-10-1 en utilisant l'ancien facteur de correction de paroi. Puisque les autres termes (indépendant des chambres d'ionisation) ne changent pas et ont une incertitude de $0,28 \%$, le débit de kerma dans l'air du faisceau de cobalt60 augmente de $0,09 \%$ et l'incertitude totale diminue de $0,38 \%$ à $0,31 \%$. L'utilisation des six chambres d'ionisation comme étalon primaire pour le kerma dans l'air au cobalt-60 conduirait à une valeur révisée de la comparaison LNE-LNHB/BIPM (2003) de 0,999 0(18) au lieu de 0,998 1(27).

\section{Remerciements}

Les auteurs souhaiteraient remercier Iwan Kawrakow, en ce temps membre du NRCC, pour avoir fourni un calcul d'effet de paroi de chambre avec EGSnrc pour la GCS-10-1 dans un faisceau de cobalt-60.

\section{Références}

[1] Allisy-Roberts P.J., Burns D.T. et Kessler C., "Summary of the BIPM.RI(I)-K1 comparison for air kerma in 60Co gamma radiation", Final report for BIPM.RI(I)-K1 2007-10-30, Metrologia, 44, 2007, 06006.

[2] Delaunay F., et al., "New LNHB primary standard for ${ }^{60}$ Co air kerma", Metrologia, 47, 2010, 652-658

[3] BÜERMANN L. et BURNS D.T., "Air-kerma cavity standards", Metrologia, 46, 2009, S24-S38.

[4] Allisy-Roberts P.J., Kessler C., Burns D.T., DELAUNAY F. et LEROY E., "Comparison of the standards for air kerma of the LNE-LNHB and the BIPM for ${ }^{60}$ Co gamma radiation", Rapport BIPM, 06/02, 2006, disponible sur: http://www.bipm.org/utils/common/pdf/ rapportBIPM/2006/02.pdf.

[5] ICRU report 31, "Average energy required to produce air", International commission on radiation units and measurements, 1979.

[6] BOAG J.W., "Ionization chambers", The dosimetry of ionizing radiation - volume II, Academic Press Inc., 1987.

[7] NiATEL M.T., "An experimental study of ion recombination in parallel-plate free-air ionization chambers", Phys. Med. Biol., 12, 1967, 555-563.

[8] BÉ M.M., ChISTÉ V. et Dulieu C., "Radioactive halflives - Table of recommended values", 2006, disponible sur: http://www.nucleide.org/NucData.htm. 
[9] BiELAJEW A.F., "An analytic theory of the point-source non-uniformity correction factor for thick-walled ionisation chambers in photon beams", Phys. Med. Biol., 35, 1990, 517-538.

[10] BielaJew A.F et Rogers D.W.O., "Implications of new correction factors on primary air kerma standards in ${ }^{60} \mathrm{Co}-$ beams", Phys. Med. Biol., 37, 1992, 1283-1291.

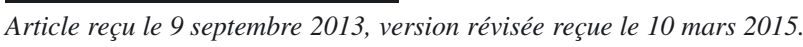

[11] KaWraKow I. et Rogers D.W.O., "The EGSnrc code system: Monte Carlo simulation of electron and photon transport." NRCC report PIRS-701, 2002.

[12] Salvat F., Fernandez-Varea J.M., Acosta E. et SEMPAU J., "PENELOPE - A code system for Monte Carlo simulation of Electron and Photon transport", NEA/NSC/DOC, 2001, 19 Report, ISBN 92-64-18475.

[13] BIELAJEW A.F., "Ionisation cavity theory: a formal derivation of perturbation factors for thick-walled ion chambers in photon beams", Phys. Med. Biol., 31, 2, 1986, 161-170. 